\title{
The captopril challenge test for diagnosing primary Aldosteronism in a Chinese population
}

\author{
Ke-ying Zhu ${ }^{1 \dagger}$, Yan Zhang ${ }^{2 \dagger}$, Wen-jing Zhang ${ }^{1}$, Hong-yun $\mathrm{Li}^{1}$, Wen-huan Feng ${ }^{1}$, Da-long Zhu ${ }^{1,2^{*}}$ and Ping $\mathrm{Li}^{1^{*}}$
}

\begin{abstract}
Background: The Captopril challenge test (CCT) is an easy-conduct confirmatory test for diagnosing primary aldosteronism (PA). Guidelines show that plasma aldosterone is normally suppressed by captopril $(>30 \%)$ in primary hypertension (PH) and in healthy people. It is unclear whether this standard is applicable in Chinese subjects. The aim of the present study was to investigate the post-CCT efficacy of plasma aldosterone concentration (PAC) suppression and determine the post-CCT aldosterone renin activity ratio (ARR) and PAC for PA diagnosis.

Methods: We recruited 110 consecutive patients with PA, 163 with primary hypertension (PH), and 40 healthy volunteers (NC). The CCT was conducted in all patients. Total sodium intake was estimated from 24-h urinary excretions. ROC curves were used to analyze the efficiency of different CCT diagnostic criteria for diagnosing PA.

Results: In NC and PH patients, PRA was increased and PAC was decreased post-CCT $(P<0.05)$. The mean degree of PAC decline after CCT was approximately $9.3 \%$, and only $11.7 \%$ of the patients with PH showed a greater than $30 \%$ suppression of PAC after CCT. In patients with PA, the post-CCT change in PRA and PRC was slight. The postCCT degree of PAC decline was unrelated to dietary salt intake. The areas under the ROC for the post-CCT ARR, PAC and PAC suppression \% were $0.994,0.754$ and 0.606 , respectively. The optimal post-CCT cutoff value for ARR for diagnosing PA was 20, which yielded a sensitivity and specificity of 94.0 and $99.4 \%$, respectively.

Conclusions: The PAC suppression percentage after CCT recommended by current clinical guidelines is not applicable when diagnosing Chinese subjects with PA. Compared to post-CCT PAC, post-CCT ARR was a better approach, having an optimal cutoff of 20 when interpreting the results of the CCT in Chinese patients. We found no relationship between high salt intake and low responses of the renin-angiotensin system (RAS) to the CCT.
\end{abstract}

Keywords: Primary aldosteronism, Diagnosis, Captopril challenge test

\section{Background}

Primary aldosteronism (PA) is the most common cause of secondary hypertension and affects approximately $10 \%$ of patients with essential hypertension [1]. In resistant hypertensive cohorts, the prevalence of PA is approximately $20 \%$ [2]. In addition to its high prevalence, patients with PA have higher cardiovascular morbidity and mortality than are reported in patients with essential hypertension and the same degree of blood pressure (BP) elevation $[3,4]$. Furthermore, specific treatments are available

\footnotetext{
* Correspondence: zhudalong@nju.edu.cn; li78321@yeah.net ${ }^{\dagger}$ Ke-ying Zhu and Yan Zhang contributed equally to this work. 'Department of Endocrinology, Drum Tower Hospital Affiliated to Nanjing University Medical School, Nanjing 210008, People's Republic of China Full list of author information is available at the end of the article
}

that can cure hypertension and ameliorate its complications [5]. Therefore, early and accurate diagnosis is imperative.

A previous study demonstrated that in China, the overall prevalence rate of PA in adults with resistant hypertension was $7.1 \%$ [6], which is not the highest among those previously reported in other ethnic populations outside China [7, 8]. However, China has a large general population with a prevalence of hypertension of $29.6 \%$ [9]. It is challenging to accurately and quickly diagnose PA in such a large population. Hence, convenient diagnostic processes with a high efficiency for identifying PA are currently urgently needed in China.

(c) The Author(s). 2019 Open Access This article is distributed under the terms of the Creative Commons Attribution 4.0 International License (http://creativecommons.org/licenses/by/4.0/), which permits unrestricted use, distribution, and reproduction in any medium, provided you give appropriate credit to the original author(s) and the source, provide a link to the Creative Commons license, and indicate if changes were made. The Creative Commons Public Domain Dedication waiver (http://creativecommons.org/publicdomain/zero/1.0/) applies to the data made available in this article, unless otherwise stated. 
Confirmation tests are a crucial step in the process of obtaining a functional diagnosis of PA. The Endocrine Society's guidelines for PA recommend 4 confirmatory tests, including the fludrocortisone suppression test (FST), saline infusion test (SIT), captopril challenge test $(\mathrm{CCT})$, and oral sodium loading. Among these confirmatory tests [1], the CCT is easy-to-conduct and is considered to have the same diagnostic efficiency as the other tests [1]. However, there is no consensus on how the results of the CCT should be interpreted $[1,10,11]$. The Endocrine Society guidelines recommend that a postCCT suppression percentage $<30 \%$ confirms PA, but this threshold has not been verified in studies [1]. However, other groups prefer to use the post-CCT aldosterone renin activity ratio (ARR) or post-CCT plasma aldosterone concentration (PAC) to diagnose PA [12-15]. Diets and genetic susceptibilities are different between Chinese and Western populations [16], and previous studies have suggested that Chinese and Western individuals perform differently on the SIT [17]. Therefore, the best approach to using and cutoff value for the CCT in Chinese patients have not yet been defined.

The present study aimed to investigate the usefulness of, optimal interpretation approach to, and cutoff value for the CCT in diagnosing PA in Chinese patients. Furthermore, the correlation between estimated sodium intake and CCT performance was also assessed.

\section{Methods}

\section{Subjects}

The present study was conducted in the Nanjing Drum Tower Hospital. All subjects provided informed consent. We retrieved data from hypertensive patients who were referred to our unit from January 2011 to December 2016 with a suspicion of PA because of resistant hypertension, spontaneous or diuretic-induced hypokalemia, or the finding of an adrenal mass. Potassium-wasting or -sparing diuretics were discontinued at least 4 weeks prior to testing. Other drugs, including $\beta$-blockers, angiotensin-converting enzyme inhibitors and angiotensin receptor blockers, were withdrawn for at least 2 weeks. Patients with severe hypertension were prescribed $\alpha$-blockers, and long-acting calcium channel blockers were prescribed if necessary. Dietary sodium intake was unrestricted. Patients with hypokalemia were given adequate oral or intravenous potassium supplements to maintain serum potassium levels higher than $3.0 \mathrm{mmol} / \mathrm{L}$ before the study was begun.

Data representing normal controls were retrieved from healthy volunteers who participated in the study and had a BP below 140/90 $\mathrm{mmHg}$ and no history of hypertension and were not receiving any medication. Finally, we included 110 PA patients, 163 primary hypertension (PH) patients and 40 normal controls (NC).

\section{Biochemical measurements}

Sodium and potassium levels in blood and urine were measured using fully automated instrumentation. Plasma renin activity (PRA) was measured by radioimmunoassay using a commercial kit (Atomic hi-tech Co., LTD, Beijing, China). The intra- and inter-assay coefficients of variation (CVs) for PRA were 10 and 15\%, respectively. PAC was measured by radioimmunoassay with commercial kits (Northern Technical and Biological Institute, Beijing, China). The intra-assay and inter-assay CVs for this assay were 5.6 and $6.2 \%$, respectively.

\section{Diagnostic procedures}

Blood samples for PRA and PAC were obtained in all patients between 0800 and $0900 \mathrm{~h}$ after overnight fasting and at least $2 \mathrm{~h}$ lying in a supine position and then again after $2 \mathrm{~h}$ of keeping in an upright position. The CCT was performed in the morning. Patients received $50 \mathrm{mg}$ of captopril orally after they were upright (sitting, standing, or walking) for at least $2 \mathrm{~h}$ and seated for $5-15 \mathrm{~min}$. Blood samples were drawn to measure PRA and PA at time 0 and at $2 \mathrm{~h}$ after challenge. The patient remained seated during this period.

All hypertensive patients underwent an enhanced adrenal computed tomography (CT) scan with fine cuts $(3 \mathrm{~mm})$. Adrenal vein sampling (AVS) procedures were performed between 0800 and $1200 \mathrm{~h}$ by one radiologist using the bilateral simultaneous technique without cosyntropin stimulation. According to previous recommendations, successful cannulation was considered a selectivity index $(\mathrm{SI}) \geq 2$, and lateralization of aldosterone secretion was defined as a lateralization index $(\mathrm{LI}) \geq 2$.

$\mathrm{PH}$ was defined as a systolic and diastolic BP greater than $140 / 90 \mathrm{mmHg}$ according to the established criteria and excluding secondary hypertension, such as renal parenchymal hypertension, renovascular hypertension, endocrine hypertension, aortic coarctation, sleep apnea syndrome, and drug-induced hypertension.

Identification of PA required all of the following criteria: (1) ARR $\geq 25$ and $\mathrm{PAC}>12 \mathrm{ng} / \mathrm{dL}$ in addition to (2) at least one of the following additional endocrine alterations: a. upright $\mathrm{PRA}<1.0 \mathrm{ng} / \mathrm{ml} / \mathrm{h}$, b. ARR postCCT $\geq 20$ or post-CCT PAC suppressed less than $30 \%$.

Identification of aldosterone-producing adenoma (APA) was based on the following criteria: (1) evidence of PA as defined above, (2) lateralization of aldosterone secretion at CT and/or AVS, (3) evidence of adenoma at $\mathrm{CT}$ and/or surgery and/or pathology, and (4) demonstration of normokalemia and hypertension cure or improvement or normalization of PAC and PRA at follow-up after adrenalectomy.

Identification of idiopathic hyperaldosteronism (IHA) was based on the following criteria: (1) evidence of PA as defined above, (2) bilateral adrenal lesions on CT scan 
and/or aldosterone secretion at AVS, (3) demonstration of normokalemia and improvement of hypertension after treatment with mineralocorticoid receptor antagonists.

\section{Statistical analysis}

Normally distributed data are expressed as the means \pm standard deviation, and abnormally distributed data are expressed as a median (interquartile intervals 25-75\%). Values between groups were compared by one-way analysis of variance for parametric data and the Kruskall-Wallis test for nonparametric data. The diagnostic accuracy of post-CCT PAC suppression (as a percentage), PAC and ARR for identifying PA was assessed with a receiver-operated characteristic (ROC) curve and the area under the ROC curve (AUC). The best cutoff and both sensitivity and specificity are provided. A value of $P<0.05$ was considered to be statistically significant. Most statistical analyses were performed using the software package SPSS 21.0 for Microsoft Windows (SPSS Inc.). The ROC curve comparison was performed with MedCalc software 15.2.2 (MedCalc Software).

\section{Results}

\section{Clinical characteristics of patients with PA}

We recruited 110 consecutive patients with PA. Of these patients, 82 had an APA, and 28 had IHA. The clinical characteristics of each group are summarized in Table 1. There was no significant difference among the groups in age, sex and BMI. Systolic blood pressure (SBP) and diastolic blood pressure (DBP) were higher in the PA and $\mathrm{PH}$ groups than in the NC group $(P<0.01)$. BP was higher in the APA group than in the PH group $(P<0.01)$. Compared to the $\mathrm{NC}$ and $\mathrm{PH}$ groups, patients in the PA group had lower serum potassium and higher serum sodium and urine potassium levels $(P<0.01)$. PAC was markedly higher and PRA was significantly lower in the $\mathrm{PA}$ group than in the $\mathrm{PH}$ and $\mathrm{NC}$ groups $(P<0.01)$. The ARR was consequently much higher in the PA group than in the $\mathrm{PH}$ and $\mathrm{NC}$ groups $(P<0.05)$. The prevalence of hypokalemia was higher in the APA group than in the IHA group (92.7\% vs $75 \%$ ). Compared to patients with IHA, those with APA had higher PAC $(P<0.01)$. No other significant differences were detected between the APA and IHA groups.

\section{Captopril challenge test in the normal control and patient groups}

In the $\mathrm{NC}$ and $\mathrm{PH}$ groups, PRA was higher and PAC was lower after CCT $(\mathrm{P}<0.05)$. As a result, ARR was lower post-CCT than pre-CCT $(P<0.01)$. However, the mean post-CCT suppression percentage of $\mathrm{PAC}$ was only approximately $9.3 \%$, and only $11.7 \%$ of the patients with $\mathrm{PH}$ showed a post-CCT decrease in PAC greater than $30 \%$. In PA patients, the CCT increased PRA and lowered PAC slightly. After CCT, PAC remained higher and PRA was lower in the PA group than in the PH and NC groups $(P<0.01)$. As a result, ARR remained higher post-CCT higher in the APA and IHA groups than in the $\mathrm{PH}$ and NC groups $(P<0.01)$. The post-CCT PAC was still higher in the APA group than in the IHA group $(P<0.05)$. No other differences were detected in the indexes between the APA and IHA groups after CCT (Table 2).

\section{Effects of sodium intake on CCT performance}

We divided our subjects according to daily urinary sodium excretion to investigate the effect of sodium intake on CCT performance. Patients were divided into

Table 1 Clinical characteristics of the included normal controls and patients

\begin{tabular}{lllll}
\hline & $\mathrm{NC}(n=40)$ & $\mathrm{PH}(n=163)$ & $\mathrm{IHA}(n=28)$ & APA $(n=82)$ \\
\hline Age $(\mathrm{y})$ & $49.0 \pm 11.5$ & $48.6 \pm 15.4$ & $51.5 \pm 10.9$ & $46.0 \pm 12.9$ \\
Sex $(\mathrm{M} / \mathrm{F})$ & $19 / 21$ & $92 / 71$ & $18 / 10$ & $37 / 45$ \\
$\mathrm{BMI}\left(\mathrm{kg} / \mathrm{m}^{2}\right)$ & $25.0 \pm 3.6$ & $25.6 \pm 4.0$ & $25.2 \pm 2.4$ & $25.5 \pm 8.9$ \\
SBP $(\mathrm{mmHg})$ & $124.2 \pm 9.3$ & $145.4 \pm 19.6^{\mathrm{A}}$ & $144.5 \pm 13.4^{\mathrm{A}}$ & $152.0 \pm 208^{\mathrm{AB}}$ \\
DBP $(\mathrm{mmHg})$ & $75.1 \pm 9.5$ & $89.0 \pm 14.4^{\mathrm{A}}$ & $90.3 \pm 11.9^{\mathrm{A}}$ & $94.7 \pm 15.3^{\mathrm{AB}}$ \\
Prevalence of hypokalemia (\%) & - & - & 75.0 & $92.7^{\mathrm{C}}$ \\
Serum potassium (mmol/L) & $4.0 \pm 0.29$ & $4.0 \pm 0.25$ & $3.3 \pm 0.45^{\mathrm{AB}}$ & $3.2 \pm 0.53^{\mathrm{AB}}$ \\
Serum sodium (mmol/L) & $141.1 \pm 1.9$ & $141.0 \pm 1.8$ & $143.2 \pm 2.3^{\mathrm{AB}}$ & $143.5 \pm 3.7^{\mathrm{AB}}$ \\
Urine potassium (mmol/24h) & $36.7 \pm 12.9$ & $38.9 \pm 15.6$ & $57.5 \pm 27.0^{\mathrm{AB}}$ & $58.2 \pm 26.8^{\mathrm{AB}}$ \\
Supine PRA (ng/ml.h) & $1.0(0.55 \sim 1.9)$ & $1.4(0.65 \sim 2.4)$ & $0.11(0.03 \sim 0.26)^{\mathrm{AB}}$ & $0.14(0.025 \sim 0.33)^{\mathrm{AB}}$ \\
Supine PAC (ng/dl) & $11.2 \pm 4.7$ & $12.7 \pm 3.8$ & $15.0 \pm 6.1^{\mathrm{Ab}}$ & $17.8 \pm 5.5^{\mathrm{ABC}}$ \\
ARR & $10.6(5.4 \sim 17.1)$ & $8.9(5.4 \sim 17.5)$ & $84.6(64.6 \sim 556.2)^{\mathrm{AB}}$ & $117.5(49.4 \sim 695.8)^{\mathrm{AB}}$ \\
\hline Data & & &
\end{tabular}

Data are expressed as the mean \pm SD or median (25th-75th percentiles). NC normal controls, $P H$ primary hypertension, IHA idiopathic hyperaldosteronism, $A P A$ aldosterone-producing adenoma, $B M I$ body mass index, $S B P$ systolic blood pressure, $D B P$ diastolic blood pressure, $P R A$ plasma renin activity, $P A C$ plasma aldosterone concentration, $A R R$ aldosterone to renin ratio. ${ }^{\mathrm{A}} P<0.01,{ }^{\mathrm{a}} P<0.05$ vs. $\mathrm{NC} ;{ }^{\mathrm{B}} P<0.01,{ }^{\mathrm{b}} P<0.05$ vs. $\mathrm{PH} ;{ }^{\mathrm{C}} P<0.01,{ }^{\mathrm{C}} P<0.05$ vs. IHA 
Table 2 Results of the captopril challenge test $(C C T)$ in normal controls and patients

\begin{tabular}{|c|c|c|c|c|c|c|c|}
\hline & \multicolumn{3}{|l|}{ Pre-CCT } & \multicolumn{3}{|l|}{ Post-CCT } & \multirow{2}{*}{$\begin{array}{l}\text { PAC } \\
\text { suppression } \\
\text { percentage (\%) }\end{array}$} \\
\hline & PRA (ng/ml.h) & PAC (ng/dl) & ARR & PRA (ng/ml.h) & PAC (ng/dl) & ARR & \\
\hline $\mathrm{NC}(n=40)$ & $1.4(0.91 \sim 4.2)$ & $13.2 \pm 4.7$ & $6.9(3.9 \sim 14.4)$ & $3.0(1.6 \sim 5.3) *$ & $12.1 \pm 4.4 \dagger$ & $3.9(1.8 \sim 7.3) \dagger$ & $10.2(-2.6 \sim 20.8$ \\
\hline $\mathrm{PH}(\mathrm{n}=163)$ & $2.3(1.2 \sim 3.6)$ & $13.6 \pm 3.9$ & $6.1(3.5 \sim 10.2)$ & $3.3(1.8 \sim 5.7) \dagger$ & $12.2 \pm 4.1 \dagger$ & $3.4(2.2 \sim 7.0) \dagger$ & $10.6(-1.4 \sim 21.5$ \\
\hline$A(n=28)$ & $0.23(0.05 \sim 0.36)^{\mathrm{AB}}$ & $15.5 \pm 6.4^{\mathrm{ab}}$ & $60.0(34.8 \sim 169.2)^{\mathrm{AB}}$ & $0.31(0.20 \sim 0.58)^{\mathrm{AB}}+$ & $14.9 \pm 5.6^{\mathrm{aB}}$ & $41.1(27.9 \sim 61.2)^{\mathrm{AB}}$ & $3.4(-15.1 \sim 17.1)$ \\
\hline $\operatorname{APA}(n=82)$ & $0.21(0.048 \sim 0.35)^{\mathrm{AB}}$ & $18.2 \pm 4.9^{\mathrm{ABC}}$ & $80.0(47.7 \sim 263.9)^{\mathrm{AB}}$ & $0.30(0.080 \sim 0.53)^{\mathrm{AB}}+$ & $17.4 \pm 5.0^{\mathrm{ABC}}+$ & $54.7(35.2 \sim 196.9)^{\mathrm{AB}}$ & $3.8(-5.3 \sim 11.7)^{\mathrm{b}}$ \\
\hline
\end{tabular}

Data are expressed as the mean \pm SD or median (25th-75th percentiles). ${ }^{A} P<0.01,{ }^{a} P<0.05$ vs. NC; ${ }^{B} P<0.01,{ }^{b} P<0.05$ vs. $P H ;{ }^{C} P<0.01,{ }^{C} P<0.05$ vs. IHA; ${ }^{*} P<0.05$, $† P<0.01$ vs pre-CCT in each group

low and high sodium intake groups according to median daily urinary sodium. There were no significant differences between the PA and $\mathrm{PH}$ patient groups and the NC group in either pre- or post-CCT PAC, PRA and ARR values. Neither the PAC suppression percentage nor the PRA incretion percentage differed among the PA, PH and NC groups (Table 3).

\section{Diagnostic accuracy of the CCT}

According to the Endocrine Society Clinical Practice Guidelines, a post-CCT PAC suppression percentage less than $30 \%$ is recommended to confirm PA. ${ }^{1}$ Other association guidelines recommend the use of post-CCT ARR or PAC for diagnosing PA $[10,11]$. Using ROC curves, we investigated the efficiency of different diagnostic criteria in the CCT for diagnosing PA. The AUC of the PAC suppression percentage was only 0.606, which suggests that the post-CCT PAC suppression percentage is of no value for diagnosing PA in our population. However, when the post-CCT ARR or PAC was used, the AUC of the CCT increased to 0.994 (95\% CI, $0.987-1.000, P<0.001)$ and 0.754 (95\% CI, 0.699$0.809, P<0.001)$, respectively. The AUC of the postCCT ARR was larger than that of the post-CCT PAC $(P<0.01)$ (Fig. 1). The optimal cutoff value for the post-CCT ARR for diagnosing PA was 20, with a sensitivity and specificity of $94.0 \%$ (95\% CI, 88.58-
97.39\%) and 99.4\% (95\% CI, 96.63-99.98\%), respectively. The optimal cutoff for the post-CCT PAC was set at 13 $\mathrm{ng} / \mathrm{dL}$, which yielded a sensitivity and specificity of $70.1 \%$ (95\% CI, 61.64-77.74\%) and 72.4\% (95\% CI, 64.86-79.1\%), respectively. These values were lower than those for the post-CCT ARR $(P<0.001)$.

\section{Discussion}

Four testing procedures (oral sodium loading, SIT, FST, and $\mathrm{CCT}$ ) are recommended by the Endocrine Society's 2008 and 2016 clinical guidelines for PA confirmation diagnosis $[1,18]$. Until recently, insufficient direct evidence was available for recommending any one of these tests over all others. The choice of confirmatory test for PA diagnosis is commonly determined after considering cost, patient compliance, routine laboratory tests, and local expertise, as suggested by the guidelines [1]. Because it is safe, cost-effective, and feasible to perform, the CCT is widely used for PA diagnosis in China. The results of the current study reveal that the post-CCT degree of PAC suppression below 30\% recommended by the Endocrine Society is ineffective in diagnosing PA in Chinese patients with hypertension. Compared to postCCT PAC, post-CCT ARR is a more reliable metric for diagnosing PA in Chinese populations.

The diagnostic efficiency of the CCT varies substantially among previous studies [12-15, 19-21]. This may

Table 3 Results of the CCT according to median $\mathrm{Na}^{+}$Intake

\begin{tabular}{|c|c|c|c|c|}
\hline & \multicolumn{2}{|l|}{ APA and IHA } & \multicolumn{2}{|l|}{$\mathrm{PH}$ and $\mathrm{NC}$} \\
\hline & Below median $(n=54)$ & Above median $(n=53)$ & Below median $(n=102)$ & Above median $(n=101)$ \\
\hline Urine sodium (mmol/24 h) & $106.3 \pm 30.0$ & $247.6 \pm 76.7^{A}$ & $109.4 \pm 27.7$ & $205.5 \pm 41.5^{\mathrm{A}}$ \\
\hline Pre-PAC (ng/dl) & $17.7 \pm 5.9$ & $17.4 \pm 5.0$ & $13.6 \pm 4.0$ & $13.4 \pm 4.2$ \\
\hline Pre-PRA (ng/ml.h) & $0.20(0.048 \sim 0.42)$ & $0.23(0.065 \sim 0.35)$ & $2.2(0.96 \sim 3.7)$ & $2.3(1.2 \sim 3.7)$ \\
\hline Pre-ARR & $63.1(45.1 \sim 300.4)$ & $81.5(48.0 \sim 165.2)$ & $6.8(3.7 \sim 13.1)$ & $5.9(3.5 \sim 10.1)$ \\
\hline Post-PAC (ng/dl) & $17.2 \pm 5.7$ & $16.4 \pm 5.0$ & $12.1 \pm 4.0$ & $12.2 \pm 4.3$ \\
\hline Post-PRA (ng/ml.h) & $0.28(0.050 \sim 0.62)$ & $0.31(0.18 \sim 0.49)$ & $3.4(1.6 \sim 6.1)$ & $3.0(1.8 \sim 5.0)$ \\
\hline Post-ARR & $50.1(28.5 \sim 223.3)$ & $52.0(32.1 \sim 89.9)$ & $3.1(1.9 \sim 7.2)$ & $3.7(2.2 \sim 6.9)$ \\
\hline PAC suppression percentage (\%) & $3.7(-8.1 \sim 11.8)$ & $3.4(-6.1 \sim 13.3)$ & $12.9(-0.73 \sim 20.3)$ & $7.9(-3.0 \sim 21.9)$ \\
\hline PRA incretion percentage (\%) & $25.1(-21.3 \sim 102.3)$ & $50.5(0.0 \sim 129.0)$ & $55.0(9.3 \sim 158.0)$ & $53.0(10.1 \sim 112.1)$ \\
\hline
\end{tabular}

${ }^{A} P<0.01$ vs. below median 


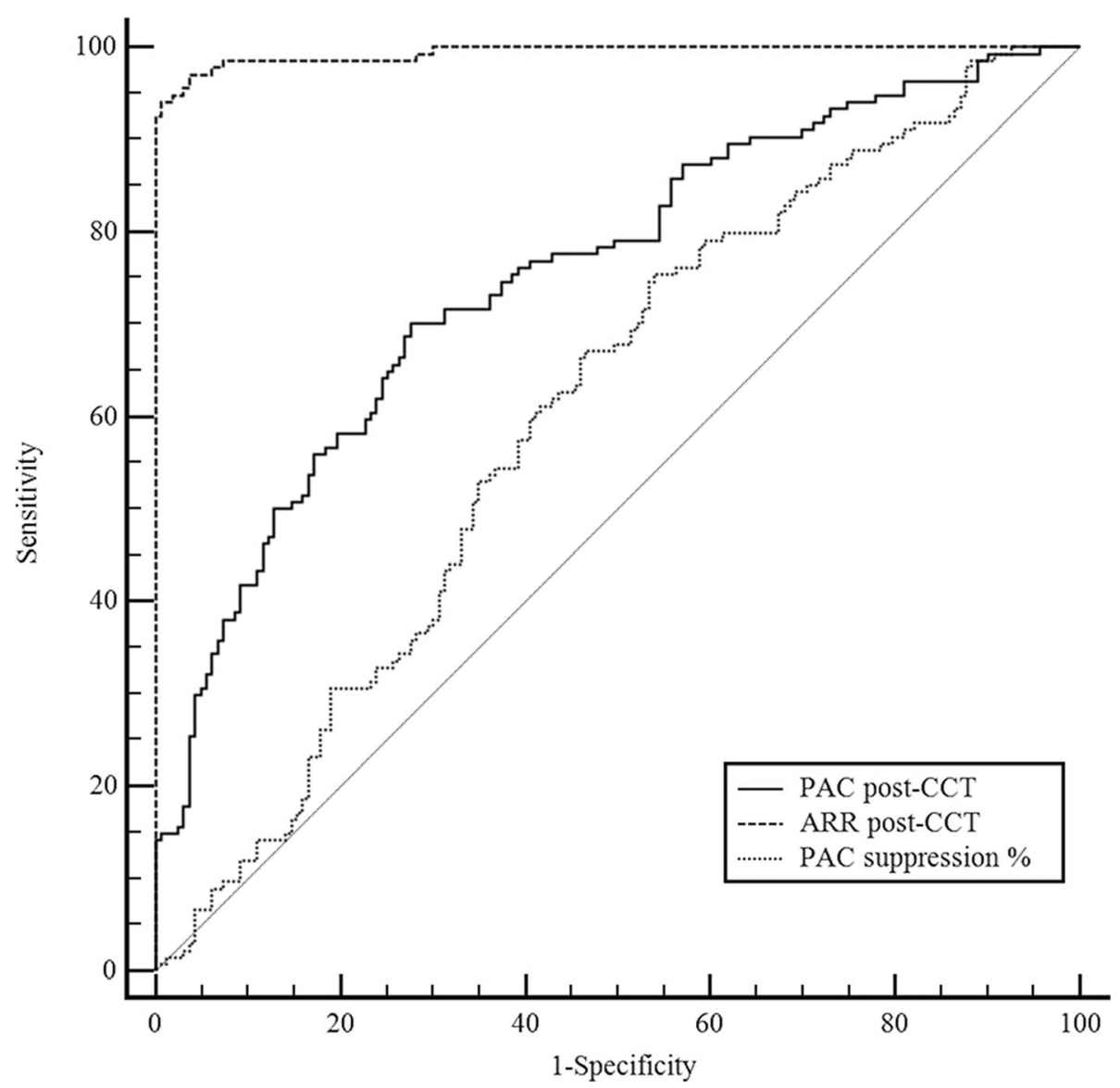

Fig. 1 ROC curve analysis of 3 diagnostic criteria in the CCT used for PA diagnosis. PAC suppression \%: plasma aldosterone concentration suppression percentage in the captopril challenge test, PAC post-CCT: plasma aldosterone concentration after the captopril challenge test, ARR post-CCT: aldosterone-renin ratio after the captopril challenge test

be because of differences in the doses of captopril ( 25 or $50 \mathrm{mg}$ ), in the time point for blood draws (i.e., 60, 90, or 120 min after captopril administration), in the method used to interpret the results (i.e., PAC suppression percentage, post-CCT PAC, or post-CCT ARR), and in the criteria used to diagnose PA. The Endocrine Society's clinical guidelines concluded that CCT has the same diagnostic efficiency as other confirmation tests and suggested using the PAC suppression percentage as a clinical diagnostic approach. PA is diagnosed by CCT when the PAC is not suppressed by captopril $(<30 \%)[1,18]$. The results of the present study showed, after CCT, the PAC decreased by $3.8 \%$ in the APA group, $3.4 \%$ in the IHA group, $10.6 \%$ in the PH group and $10.2 \%$ in the NC group. These differences in the percentage suppression of the PAC among the four groups were not significant. These findings indicate that using a suppression percentage of $30 \%$ as the approach cutoff may cause many hypertensive patients to be misdiagnosed. Two recently published studies support our results. Both found that the percentage suppression in the PAC after CCT was low in Chinese patients [22, 23]. Given that our study included patients recruited from areas of China different from those in the other two studies, our data provide additional new evidence supporting the conclusion that using a post-CCT PAC suppression percentage $>30 \%$ as a criteria is not appropriate for diagnosing PA in Chinese patients.

In addition to our study, other data collected in China have demonstrated that PAC suppression in the CCT or SIT is lower in Chinese than in Western patients $[17,22,23]$. The mechanism underlying this variation between Chinese and Western populations has not been clarified. A high salt intake has been postulated to inhibit renin-angiotensin system activity and to thereby alter the results of confirmation tests used to diagnose PA [24-26]. Rossi et al. showed that the accuracy of the CCT for PA diagnosis was affected by sodium intake. The optimal aldosterone cutoff value for PA diagnosis was elevated in individuals with a higher median daily sodium urinary excretion [15]. The Chinese diet is high in sodium. Our and other studies 
performed in China have demonstrated that 24-h urine sodium excretion (widely used to estimate daily sodium consumption) is higher in China than in Western countries $[16,27]$. It is logical to speculate that a long-term high salt diet might be one of the factors that contributes to the lower response to the CCT observed in Chinese subjects. However, when we split our patients according to the median daily urinary sodium, we found that plasma aldosterone and PRA values were not different between low- and high-sodium intake patients. Li et al. confirmed the same phenomenon in their study [17]. They found no evidence that the lower response of the renin-angiotensin system (RAS) to the SIT was related to high-salt intake [17]. Some possible explanations for the fact that sodium intake had no effect on RAS activity in our and other studies include the following: 1 ) a single $24-\mathrm{h}$ urine sodium excretion measurement will not accurately reflect long-term sodium intake levels and may therefore bias the results; 2) Our and Li's studies were all conducted in hospitalized patients. It is difficult to identify differences among patients consuming almost the same relative highsodium diet. Further studies with a crossover and prospective design are needed to evaluate the effects of low- and high-sodium intake on CCT or SIT performance. In addition to sodium intake, other factors, such as genetic susceptibility, that might be related to the observed discrepancies in performance on PA confirmation tests between Chinese and Western populations should also be considered.

The principle the captopril test is based is the suppression of the serum concentration of aldosterone and an increase in the level of PRA when angiotensinconverting enzyme is inhibited in patients without autonomous aldosterone secretion [1]. Previous studies and two newly published studies performed in China have recommended using the post-CCT PAC to confirm a diagnosis of PA, but they have suggested using various cutoff values [14, 22, 23]. Other groups prefer post-CCT ARR for PA diagnosis $[12,13,15]$. The Japan and Taiwan Endocrine Society recommend the use of post-CCT PAC or post-CCT ARR for PA diagnosis [10, 11]. We compared the performance of these two approaches in our study. The results showed that post-CCT ARR was superior to post-CCT PAC (AUC $=0.994$ vs 0.754), inconsistent with the study by Song et al., who found that while post-CCT ARR is useful, post-CCT PAC has a higher sensitivity than does post-CCT ARR in PA diagnosis [22]. Our and Song's studies both detected only a small amount of post-CCT suppression in PAC but a dramatic increase in post-CCT PRA or PRC in normal and $\mathrm{PH}$ patients. Therefore, it is reasonable to suspect that post-CCT ARR is a more sensitive marker than post-CCT PAC for differentiating PA patients from normal or $\mathrm{PH}$ patients. The possible reasons for the discrepancy between our results and those of Song et al. may include the following: 1) Song et al. used FST instead of a conclusive PA diagnosis as the reference standard, and this may have biased their data interpretation; and 2) the plasma renin concentration (PRC) was measured instead of PRA in Song's study, and their ARR was different from ours.

The optimal post-CCT ARR cut-off value is variable among studies that support the use of post-captopril ARR as a PA diagnostic tool. The divergence in cutoff values may be associated with the assays and techniques used to measure aldosterone and PRA. Consistent with the guidelines of the Japan Endocrine Society, we found that the best cutoff for the post-CCT ARR was 20, which yielded relatively high sensitivity $(94.0 \%)$ and specificity (99.4\%) in this study. Therefore, we found that the postCCT ARR is a reliable marker for diagnosing PA in a Chinese population.

\section{Conclusion}

In summary, in this study, we demonstrate that CCT is a reliable functional test for confirming a PA diagnosis. Compared to Western populations, Chinese populations exhibit lower degrees of post-CCT PAC suppression. As a result, the post-CCT PAC decline below 30\% recommended as a threshold by the Endocrine Society is ineffective for diagnosing PA in Chinese hypertension subjects. Compared to the post-CCT PAC, the postCCT ARR is a better approach when used with an optimal cutoff of 20 for interpreting the results of the CCT in Chinese patients. Furthermore, there was no relationship between high salt intake and lower RAS responses in this study. Further study is needed to identify the factors underlying this variation between Chinese and Western populations.

\section{Abbreviations \\ APA: Aldosterone-producing adenoma; ARR: Aldosterone renin activity ratio; AUC: Area under the ROC curve; AVS: Adrenal vein sampling; BP: Blood pressure; CCT: Captopril challenge test; CT: Computed tomography; CVs: Coefficients of variation; DBP: Diastolic blood pressure; FST: Fludrocortisone suppression test; IHA: Idiopathic hyperaldosteronism; LI: Lateralization index; NC: Normal controls; PA: Primary aldosteronism; PAC: Plasma aldosterone concentration; $\mathrm{PH}$ : Primary hypertension; PRA: Plasma renin activity; RAS: Renin-angiotensin system; ROC: Receiver- operated characteristic; SBP: Systolic blood pressure; SI: Selectivity index; SIT: Saline infusion test}

\section{Acknowledgements}

Not applicable.

\section{Authors' contributions}

$D L Z$ and $P L$ supervised the project and approved the final version of the manuscript to be submitted. KYZ designed the research; WJZ, HYL and WHF performed all individuals' selection and data collection, $K Y Z$ and $Y Z$ drafted the initial manuscript; PL critically revised the manuscript. All of the authors read and approved the final manuscript. 


\section{Funding}

This work was supported by the Natural Science Foundation of Jiangsu Province (Grant number BK20181116) and the Project of Jiangsu Provincial Medical Youth Talent. The funding sources had no role in the design of the study, collection, analysis and interpretation of data, nor in the drafting of the manuscript.

\section{Availability of data and materials}

The datasets analyzed during the current study are available from the corresponding author on reasonable request.

\section{Ethics approval and consent to participate}

The study protocols complied with the Guidelines of the Ethics Committee of Nanjing Drum Towel Hospital. Informed consent was obtained from all subjects.

\section{Consent for publication}

Not applicable.

\section{Competing interests}

The authors declare that they have no competing interests.

\section{Author details}

${ }^{1}$ Department of Endocrinology, Drum Tower Hospital Affiliated to Nanjing University Medical School, Nanjing 210008, People's Republic of China. ${ }^{2}$ Department of Endocrinology, Drum Tower Hospital Affiliated to Nanjing Medical University School, Nanjing 210008, People's Republic of China.

Received: 27 December 2018 Accepted: 31 May 2019

Published online: 24 June 2019

\section{References}

1. Funder JW, Carey RM, Mantero F, Murad MH, Reincke M, Shibata H, Stowasser M, Young WF. The Management of Primary Aldosteronism: case detection, diagnosis, and treatment: an Endocrine Society clinical practice guideline. J Clin Endocrinol Metab. 2016;101(5):1889-916.

2. Young WF, Calhoun DA, Lenders JWM, Stowasser M, Textor SC, Young WF, Calhoun DA, Lenders JWM, Stowasser M, Textor SC. Screening for Endocrine hypertension: an Endocrine Society scientific statement. Endocr Rev. 2017;38(2):103-22

3. Milliez P, Girerd X, Plouin PF, Blacher J, Safar ME, Mourad JJ. Evidence for an increased rate of cardiovascular events in patients with primary aldosteronism. J Am Coll Cardiol. 2005;45(8):1243-8.

4. Stowasser M, Sharman J, Leano R, Gordon R, Ward G, Cowley D, Marwick T. Evidence for abnormal left ventricular structure and function in normotensive individuals with familial hyperaldosteronism type I. J Clin Endocrinol Metab. 2005;18(5):5070-6.

5. Catena C, Colussi GR, Nadalini E, Chiuch A, Gianfagna P, Sechi L. Long-term cardiac effects of adrenalectomy or mineralocorticoid antagonists in patients with primary aldosteronism. Hypertension. 2007:50(5):911-8.

6. Sang X, Jiang Y, Wang W, Yan L, Zhao J, Peng Y, Gu W, Chen G, Liu W, Ning $G$. Prevalence of and risk factors for primary aldosteronism among patients with resistant hypertension in China. J Hypertens. 2013;31(7):1465-71.

7. Rossi GP, Bernini G, Caliumi C, Desideri G, Fabris B, Ferri C, Ganzaroli C, Giacchetti G, Letizia C, Maccario M, Mallamaci F, Mannelli M, Mattarello MJ, Moretti A, Palumbo G, Parenti G, Porteri E, Semplicini A, Rizzoni D, Rossi E, Boscaro M, Pessina AC, Mantero F, Investigators PS. A prospective study of the prevalence of primary aldosteronism in 1,125 hypertensive patients. J Am Coll Cardiol. 2006:48(11):2293-300.

8. Douma S, Petidis K, Doumas M, Papaefthimiou P, Triantafyllou A, Kartali N, Papadopoulos N, Vogiatzis K, Zamboulis C. Prevalence of primary hyperaldosteronism in resistant hypertension: a retrospective observational study. Lancet. 2008;371(9628):1921-6.

9. Wang J, Zhang L, Wang F, Liu L, Wang H. Prevalence, awareness, treatment, and control of hypertension in China: results from a National Survey. Am J Hypertens. 2014;27(11):1355-61.

10. Nishikawa T, Omura M, Satoh F, Shibata H, Takahashi K, Tamura N, Tanabe A. Task force committee on primary Aldosteronism, the Japan Endocrine Society. Guidelines for the diagnosis and treatment of primary aldosteronism--the Japan Endocrine Society 2009. Endocr J. 2011;58(9):711-21.
11. Wu VC, Hu YH, Er LK, Yen RF, Chang CH, Chang YL, Lu CC, Chang CC, Lin JH, Lin YH, Wang TD, Wang CY, Tu ST, Jeff Chueh SC, Chang CC, Tseng FY, Wu KD. TAIPAI group. Case detection and diagnosis of primary aldosteronism - the consensus of Taiwan Society of Aldosteronism. J Formos Med Assoc. 2017;116(12):993-1005.

12. Wu VC, Chang HW, Liu KL, Lin YH, Chueh SC, Lin WC, Ho YL, Huang JW, Chiang CK, Yang SY, Chen YM, Wang SM, Huang KH, Hsieh BS, Wu KD. TAIPAl study group. Primary aldosteronism: diagnostic accuracy of the losartan and captopril tests. Am J Hypertens. 2009;22(8):821-7.

13. Nakama C, Kamide K, Kawai T, Hongyo K, Ito N, Onishi M, Takeya Y, Yamamoto K, Sugimoto K, Rakugi $H$. The influence of aging on the diagnosis of primary aldosteronism. Hypertens Res. 2014;37(12):1062-7.

14. Kim JH, Park KS, Hong AR, Shin CS, Kim SY, Kim SW. Diagnostic role of captopril challenge test in Korean subjects with high aldosterone-to-renin ratios. Endocrinol Metab (Seoul). 2016:31(2):277-83.

15. Rossi GP, Belfiore A, Bernini G, Desideri G, Fabris B, Ferri C, Giacchetti G, Letizia C, Maccario M, Mallamaci F, Mannelli M, Palumbo G, Rizzoni D, Rossi E, Agabiti-Rosei E, Pessina AC, Mantero F. Primary Aldosteronism prevalence in Italy study I. comparison of the captopril and the saline infusion test for excluding aldosterone-producing adenoma. Hypertension. 2007;50(2):424-31.

16. Feng W, Cai Q, Yuan W, Liu Y, Bardeesi AS, Wang J, Chen J, Huang H. Low response of renin-angiotensin system to sodium intake intervention in Chinese hypertensive patients. Medicine. 2016;95(6):e2602.

17. Li Y, Liu Y, Li J, Wang X, Yu Y. Sodium infusion test for diagnosis of primary Aldosteronism in Chinese population. J Clin Endocrinol Metab. 2016:101(1):89-95

18. Funder JW, Carey RM, Fardella C, Gomez-Sanchez CE, Mantero F, Stowasser M, Young WF Jr, Montori VM, Endocrine S. Case detection, diagnosis, and treatment of patients with primary aldosteronism: an endocrine society clinical practice guideline. J Clin Endocrinol Metab. 2008:93(9):3266-81.

19. Castro OL, Yu X, Kem DC. Diagnostic value of the post-captopril test in primary aldosteronism. Hypertension. 2002;39(4):935-8.

20. Hambling C, Jung RT, Gunn A, Browning MC, Bartlett WA. Re-evaluation of the captopril test for the diagnosis of primary hyperaldosteronism. Clin Endocrinol. 1992:36(5):499-503.

21. Westerdahl C, Bergenfelz A, Isaksson A, Valdemarsson S. Captopril suppression: limitations for confirmation of primary aldosteronism. J Renin-Angiotensin-Aldosterone Syst. 2011;12(3):326-32.

22. Song Y, Yang S, He W, Hu J, Cheng Q, Wang Y, Luo T, Ma L, Zhen Q, Zhang S, Mei M, Wang Z, Qing H, Bruemmer D, Peng B, Li Q. Chongqing primary Aldosteronism study $\mathrm{G}$. confirmatory tests for the diagnosis of primary Aldosteronism: a prospective diagnostic accuracy study. Hypertension. 2018;71(1):118-24

23. Meng $X$, Li Y, Wang $X$, Li J, Liu Y, Yu Y. Evaluation of the saline infusion test and the captopril challenge test in Chinese patients with primary Aldosteronism. J Clin Endocrinol Metab. 2018;103(3):853-60.

24. Shimosawa T. Salt, the renin angiotensin aldosterone system and resistant hypertension. Hypertension Res. 2013;36(8):657-60.

25. Koch M, Aker S, Haastert B, Rump LC. Clinical relevance of dietary salt intake on aldosterone and the aldosterone-to-renin ratio as screening parameters for primary aldosteronism. Clin Nephrol. 2010;74(3):182-9.

26. Baudrand R, Guarda FJ, Torrey J, Williams G, Vaidya A. Dietary sodium restriction increases the risk of misinterpreting mild cases of primary Aldosteronism. J Clin Endocrinol Metab. 2016;101(11):3989-96.

27. Okuda N, Stamler J, Brown IJ, Ueshima H, Miura K, Okayama A, Saitoh S, Nakagawa H, Sakata K, Yoshita K, Zhao L, Elliott P, Group IR. Individual efforts to reduce salt intake in China, Japan, UK, USA: what did people achieve? The INTERMAP population study. J Hypertens. 2014;32(12):2385-92.

\section{Publisher's Note}

Springer Nature remains neutral with regard to jurisdictional claims in published maps and institutional affiliations. 